\title{
16. THE PROPERTIES OF EXTRAGALACTIC X-RAY SOURCES FROM VISIBLE LIGHT OBSERVATIONS
}

\author{
WALLACE L. W. SARGENT \\ Hale Observatories, California Institute of Technology, Carnegie Institution of Washington, U.S.A.
}

\begin{abstract}
We describe the optical properties of the radio galaxy NGC 5128, the Seyfert galaxy NGC 4151 and the QSO 3C 273 all of which appear to be point sources of X-rays. We emphasize how the X-ray observations, particularly the low energy absorption cutoff, may help us to understand the detailed structure and source of energy in these diverse objects.

The clusters of galaxies in Virgo, Perseus, Coma and Centaurus, all associated with extended $\mathrm{X}$-ray sources are described. They have diverse shapes, central concentrations and galactic populations, but all contain a radio galaxy and, in several cases, a low frequency radio halo around it. It is concluded that the X-ray emission is likely to be non-thermal in origin.
\end{abstract}

\section{Introduction}

The remarkable observations of extragalactic X-ray sources made recently by UHURU and earlier by rocket-born detectors have added to the rich variety of phenomena manifested by the violent events in galactic nuclei. While the recent $\mathrm{X}$-ray observations have so far led to little new theoretical insight into these phenomena, it does appear that extragalactic X-ray sources can be divided into two categories - individual galaxies with active nuclei (including radio galaxies, Seyfert galaxies and quasars) and clusters of galaxies. These cluster sources appear to be extended and may themselves be due ultimately to active individual galaxies. I shall therefore divide this survey of the optical properties of the sources into two parts, dealing first with individual non-cluster sources in Section 2 and then with the clusters which contain extended sources in Section 3. The main theme will be the diversity of the objects in each category. Since some of the identifications are doubtful I shall only go into details where identification of the X-ray source is certain.

\section{Individual Galaxies With Active Nuclei}

\section{A. NGC 5128}

As Table I shows NGC 5128 (Centaurus A) is the most powerful extragalactic radio source in the sky. At a distance of $5 \mathrm{Mpc}$ it is the nearest strong radio galaxy, defined as those objects with intrinsic powers in excess of $40^{41} \mathrm{erg} \mathrm{s}^{-1}$ between $10^{8}$ and $10^{10}$ Hz. NGC 5128 appears to be a member of a loose group or chain of galaxies. The chain extends about $20^{\circ}$ or roughly $1.5 \mathrm{Mpc}$ across the southern sky (de Vaucouleurs, 1968; Arp 1968). Optically NGC 5128 is a unique object; it is shown in Figure 1. Although classified as DE 3 by Mathews et al. (1964), it differs from normal elliptical galaxies in two important respects. First it contains a broad dust lane across its center. The axis of rotation is perpendicular to this band (Burbidge and Burbidge, 
TABLE I

The strongest extragalactic radio sources

\begin{tabular}{|c|c|c|c|c|c|c|c|}
\hline Name & Other & $\begin{array}{l}\text { Radio } \\
\text { flux* }\end{array}$ & $\begin{array}{l}\text { Optical } \\
\text { magn. }\end{array}$ & $\begin{array}{l}\text { Redshift } \\
z\end{array}$ & $\begin{array}{l}\text { Optical } \\
\text { spectrum }\end{array}$ & $\begin{array}{l}\text { Type of } \\
\text { galaxy }\end{array}$ & $\begin{array}{l}\text { X-ray } \\
\text { source }\end{array}$ \\
\hline Cen A & NGC 5128 & 8700 & 7.0 & 0.0016 & w.e.; abs. & DE3 & Yes \\
\hline Cygn. A & $3 C 405$ & 8100 & 15.1 & 0.0570 & s.e. & D3 (cl) & Yes? \\
\hline Virgo A & $\begin{array}{l}3 C 274 \\
\text { (NGC 4486) }\end{array}$ & 970 & 8.7 & 0.0041 & w.e.; abs & E2 (cl) & Yes; ext. \\
\hline Pictor A & MSH 05-43 & 570 & 17.0 & 0.0342 & s.e. & ND 1 & $?$ \\
\hline Her A & $3 C 348$ & 325 & 17.0 & 0.1540 & w.e. (1 line) & D4 (cl) & Yes;? \\
\hline Fornax A & NGC 1316 & 249 & 8.9 & 0.0058 & w.e.; abs. & D4 & $?$ \\
\hline Hydra A & $3 C 218$ & 210 & 16.0 & 0.0530 & w.e. & D2 dbl. (cl) & $?$ \\
\hline $3 C 353$ & & 203 & 15.3 & 0.0307 & w.e.; abs. & D2 & $?$ \\
\hline
\end{tabular}

* Flux units at $178 \mathrm{MHz}$.

$1959,1962)$ as is the major axis of the radio source. Secondly the optical image of NGC 5128 contains faint extensions along the major axis. These faint outer parts give the impression of a spiral or even helical structure; the ends of the dust lane also curve around in a manner resembling a spiral.

Spectra of NGC 5128 show weak emission lines of [O II ], [N II ], [S II] and the Balmer lines in the vicinity of the dust band in addition to the absorption lines produced by cool stars normally found in the spectra of elliptical galaxies. The emission lines are sharp and have $\mathrm{H} \alpha$ stronger than [N II]; in those cases where weak emission lines are found in the spectra of normal ellipticals $\mathrm{H} \alpha<[\mathrm{N} \mathrm{II}]$. Burbidge and Burbidge $(1959,1962)$ found that the mean recession velocity of the gas in the center of NGC 5128 is about $100 \mathrm{~km} \mathrm{~s}^{-1}$ greater than that indicated by the stellar absorption lines; furthermore the gas appears to be rotating more rapidly than the stars. It seems plausible that in NGC 5128 the radio components were ejected along the rotation axis of the galaxy. The Burbidges suggested that the dust lane is the remnant of an outburst which produced the extended radio source and that material is now falling back into the galaxy.

Recent infrared and radio observations of NGC 5128 have indicated that one of the knots in the center of the dust lane, relatively inconspicuous on optical photographs, (Figure 1) is the nucleus of the galaxy. (Kunkel and Bradt, 1971; Wade et al., 1971; Becklin et al., 1971). The spot in question has a size of about $3^{\prime \prime} \times 5^{\prime \prime}=$ $75 \times 110 \mathrm{pc}$ and has a luminosity of about $2.4 \times 10^{41} \mathrm{erg} \mathrm{s}^{-1}$ between 0.35 and $1.0 \mu$. Becklin $e t$ al. find that the $2.2 \mu$ and $3.5 \mu$ radiation from the supposed nucleus is more strongly concentrated towards the center than the shorter wavelength radiation; they infer from this that a small non-thermal core is superimposed on a nucleus composed of stars. They note, moreover, that the central $1^{\prime \prime}$ of the nucleus has a surface brightness at $2.2 \mu 10$ times that of a similar linear distance at the center of our galaxy and 25 times that of $\mathrm{M} 31$. Wade et al. observed a weak radio component (2.9 flux units at $3.7 \mathrm{~cm}$ - negligible as compared with the radio emission from NGC 5128 as a whole) which is coincident with the infrared nucleus. This radio 


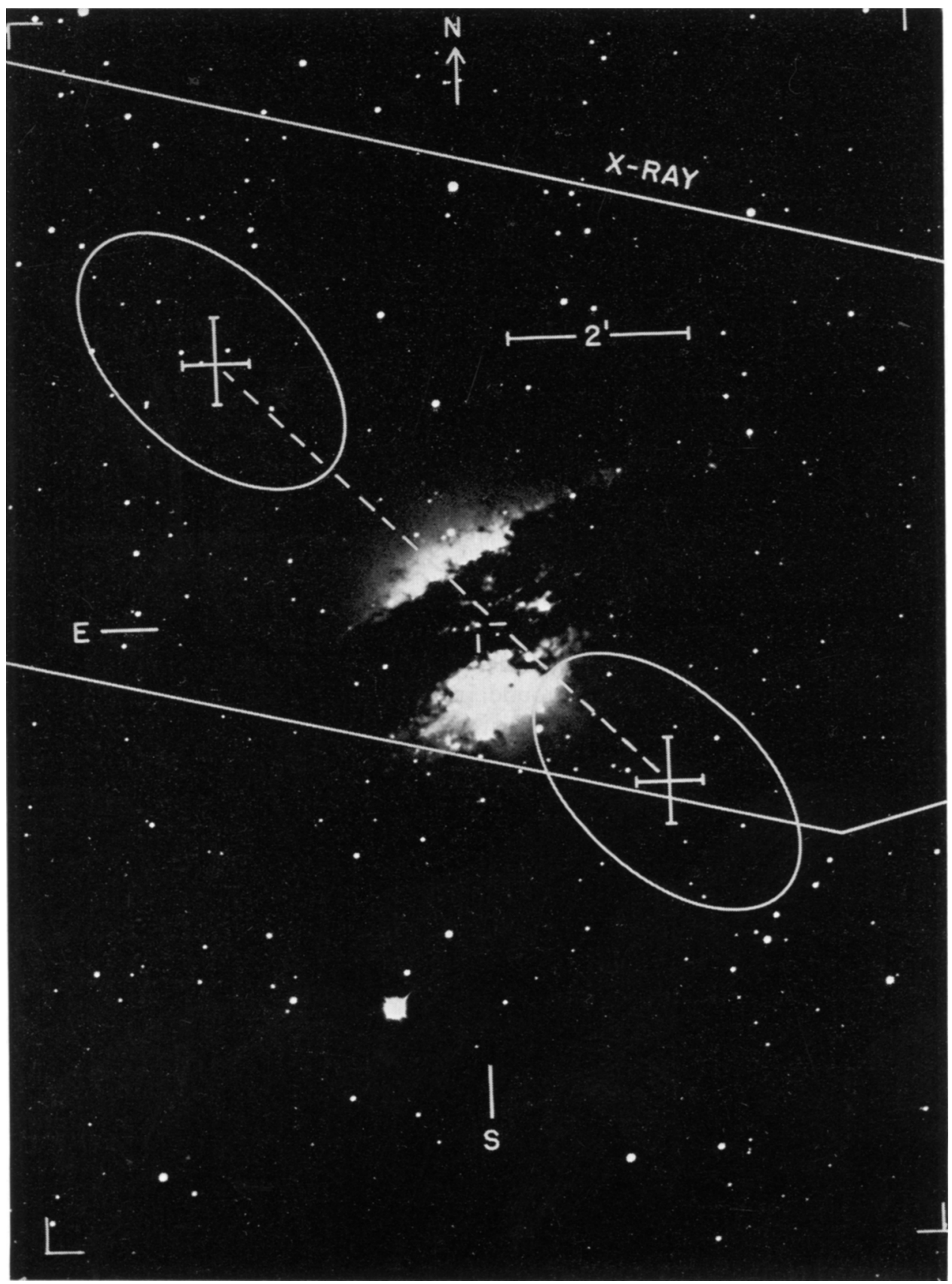

Fig. 1. NGC 5128 - Sixty-inch, IIIa-J plate taken by Dr John Graham at CTIO. The infrared hot spot, the inner radio lobes, and the $1 \sigma$ limits of a previously published UHURU X-ray error box are indicated. (From Kunkel and Bradt, 1971). 
source is inferred to have a size of less than $0.25 \mathrm{pc}$ and is similar to the nuclear sources found in the nuclei of $\mathrm{M} 87$ and other elliptical galaxies.

There is no spectroscopic evidence in the case of NGC 5128 for a hot gas which might emit thermal X-rays - as there is in the case of the nucleus of NGC 4151 . However, the UHURU measurements indicate that there is a column density of hydrogen of about $10^{23}$ atoms $\mathrm{cm}^{-2}$ in front of the X-ray source in NGC 5128. This probably indicates that the X-rays originate in the nucleus of the object because even in a spiral galaxy it would be impossible to observe such a large column density in the spiral arms. Thus the observations point to the infrared nucleus as the source of the X-ray emission; it is clearly important that further studies be made, particularly to put limits on the variability of the X-ray and infrared emission.

\section{B. NGC 4151}

This is one of the brightest Seyfert galaxies. These are mostly spiral galaxies; they contain a bright star-like nucleus which radiates a unique spectrum composed of broad emission lines (half widths from several hundred to several thousand $\mathrm{km} \mathrm{s}^{-1}$ ) superimposed on a smooth continuum which has both an ultraviolet and an infrared excess. The emission lines in Seyfert galaxies exhibit a wide range in ionization conditions - from [ $\left.\mathrm{O}_{\mathrm{I}}\right]$ to [Ne v] among the stronger lines; in this respect alone they can be distinguished spectroscopically from $\mathrm{H}$ II regions excited by hot stars. Some but not all of the N-type galaxies associated with strong radio sources have Seyfert spectra. Among the bright, nearby, Seyfert galaxies, some (for example NGC $1068 \equiv 3 C 71$ and NGC $1275 \equiv 3 C$ 84) are strong radio galaxies while others, including NGC 4151, have only a weak nuclear radio source (Wade, 1968).

NGC 4151 is probably the best studied of the Seyfert galaxies; its spectrum is illustrated in Figure 2. Table II, taken from Oke and Sargent (1968), lists most of the emission lines observable in the region $\lambda \lambda 3300-11000$ with their estimated intensities at the source assuming a distance of $10 \mathrm{Mpc}$ to NGC 4151. Iron is present as [Fe III], $[\mathrm{Fe} \mathrm{VII}]$ and $[\mathrm{Fe} \mathrm{x}]$. The coronal line $\lambda 5303$ of $[\mathrm{Fe} \mathrm{XIV}]$ is also listed in Table II; however, Weedman (1971) has recently shown that its wavelength is incorrect by a few tenths of an angstrom to be [Fe XIV].

The nucleus of NGC 4151 is unusually bright relative to the outer parts of the galaxy. The continuum between emission lines is smooth and appears to be mostly non-thermal in origin. After a contribution from hydrogen recombination has been subtracted, the remaining continuum varies as $f_{v}=K v^{-1.2}$ between $\lambda 3300$ and $\lambda 11000$. The intensity of the continuous radiation varies on a time scale of months by about 1 mag.

The emission lines in the spectrum of NGC 4151 arise in a very small region, at most $2^{\prime \prime}$ in diameter, which is only just resolved at the Earth's surface. The work of many authors (see, for example, Rees and Sargent (1972) for references) has led to the following picture of the nucleus.

(a) NGC 4151 is the prototype of those Seyfert galaxies which have broad wings to the permitted lines; the forbidden lines are sharper. It is supposed that the permitted 


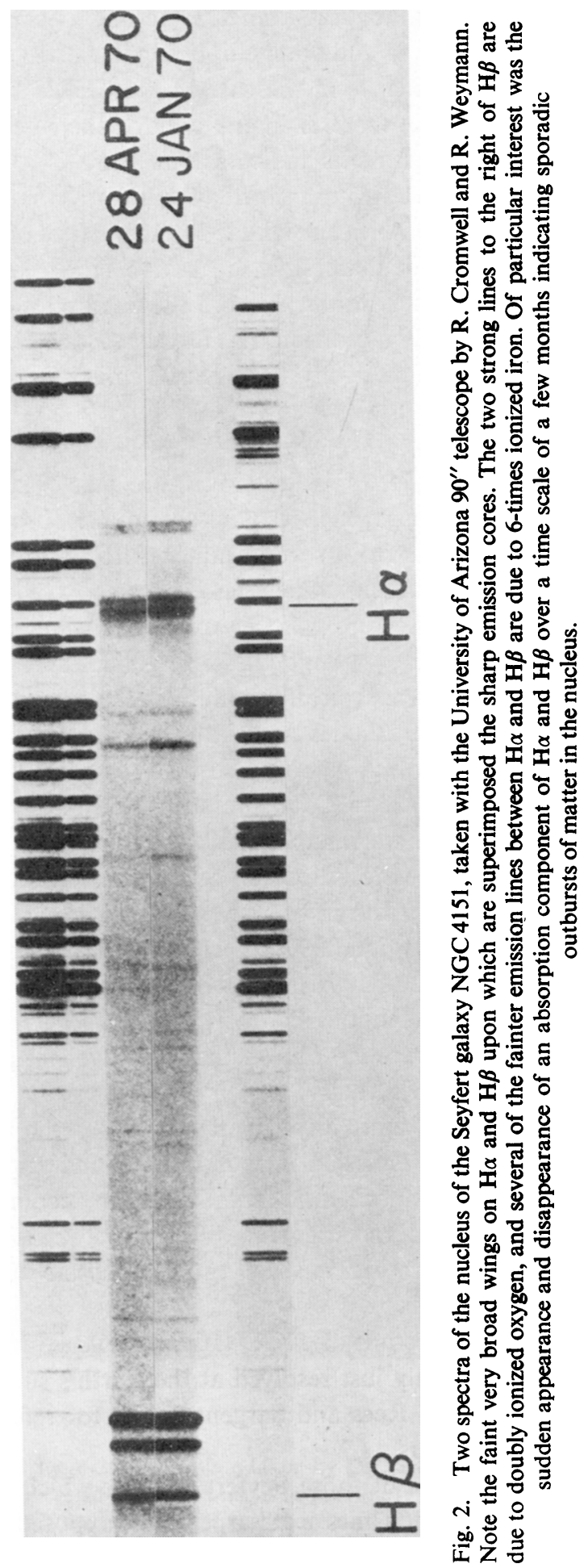


TABLE II

NGC 4151: emission-line identifications and intensities

\begin{tabular}{|c|c|c|c|c|c|}
\hline \multirow[t]{2}{*}{$\lambda(\AA)$} & \multirow[t]{2}{*}{ Ion } & \multirow{2}{*}{$\begin{array}{l}\text { Equivalent } \\
\text { width }(\AA)\end{array}$} & \multirow{2}{*}{$\begin{array}{l}\text { Flux at source } \\
\text { (units of } 10^{40} \\
\text { ergs s}^{-1} \text { ) }\end{array}$} & \multicolumn{2}{|c|}{ Strength relative to $\mathrm{H} \beta=100$} \\
\hline & & & & NGC 4151 & NGC 7027 \\
\hline 10830.2 & He I & 163.5 & 5.98 & 81 & 87 \\
\hline $\begin{array}{c}10049.4 \\
7329.9 \text { ) }\end{array}$ & $\mathrm{P} \zeta$ & 30.0: & 1.19: & 16: & 5 \\
\hline \multirow{4}{*}{$\begin{array}{l}7330.7 \\
6731.3 \\
6717.0 \\
6583.6\end{array}$} & {$[\mathrm{O} \mathrm{II}]$} & 11.0 & 0.62 & 8 & 32 \\
\hline & [S II $]$ & 33.3 & 2.08 & $28\}$ & \\
\hline & [S II] & 27.7 & 1.75 & $24\}$ & 6 \\
\hline & {$[\mathrm{N}$ II $]$} & 29.0 & 1.83 & 25 & 90 \\
\hline \multirow{2}{*}{6562.8} & $\int \mathrm{H} \alpha$ wings & 360.0 & 22.75 & $307 ?$ & 290 \\
\hline & ( $\mathrm{H} \alpha$ core & 34.0 & 2.15 & $29\}$ & 20 \\
\hline \multirow{3}{*}{$\begin{array}{l}6548.1 \\
6374.5 \\
6363.9\end{array}$} & {$[\mathrm{~N} \mathrm{II}]$} & 6.2 & 0.39 & 5 & 30 \\
\hline & {$[\mathrm{Fe} \mathrm{x}]$} & 2.8: & 0.18 & 2: & \\
\hline & [O I] & 4.7 & 0.31 & 4 & 6 \\
\hline \multirow{2}{*}{$\begin{array}{l}6300.2 \\
6085.3\end{array}$} & [O I] & 20.6 & 1.36 & 18 & 20 \\
\hline & [Fe viI $]$ & 7.6 & 0.52 & 7 & \\
\hline \multirow{2}{*}{$\begin{array}{l}5875.6 \\
5754.8\end{array}$} & He I & 4.4 & 0.31 & 4 & 11 \\
\hline & [N II] & 3.0: & $0.22:$ & 3: & 8 \\
\hline \multirow{2}{*}{$\begin{array}{l}5720.9 \\
5303.6\end{array}$} & [Fe VII] & 4.4 & 0.32 & 4 & \\
\hline & [Fe XIV] & 1.0: & 0.08 & 1: & \\
\hline \multirow{2}{*}{$\begin{array}{l}5006.8 \\
4959.9\end{array}$} & [O III] & 188.0 & 15.80 & 214 & 1460 \\
\hline & [O III] & 62.0 & 5.23 & 70 & 480 \\
\hline 4861.3 & $\int \mathrm{H} \beta$ wings & 72.0 & 6.07 & $82\}$ & 100 \\
\hline \multirow{2}{*}{$\begin{array}{l}4799.5 \\
4740.3\end{array}$} & $\begin{array}{l}\mathbf{H} \beta \text { core } \\
{[\mathrm{Fe}}\end{array}$ & $\begin{array}{r}16.0 \\
1.0:\end{array}$ & $\begin{array}{l}1.35 \\
0.10:\end{array}$ & $\begin{array}{c}18\} \\
1:\end{array}$ & 100 \\
\hline & [A IV] & 1.7 & 0.15 & 2 & 10 \\
\hline \multirow{2}{*}{$\begin{array}{l}4711.4 \\
4685.7\end{array}$} & [A IV] & 1.7 & 0.15 & 2 & 8 \\
\hline & He II & 21.7 & 1.88 & 25 & 46 \\
\hline 4658.1 & {$[\mathrm{Fe} \mathrm{III}]$} & 5.9 & 0.51 & 7 & \\
\hline \multirow{2}{*}{$\begin{array}{l}4471.5 \\
4363.2\end{array}$} & He I & 1.0: & $0.10:$ & 1: & 4 \\
\hline & [O III] & 5.4 & 0.48 & 7 & 26 \\
\hline \multirow{2}{*}{4340.5} & $\{\mathbf{H} \gamma$ wings & 21.4 & 1.92 & 26? & 47 \\
\hline & $\langle\mathbf{H} \gamma$ core & 5.6 & 0.50 & $7)$ & \\
\hline \multirow{2}{*}{$\begin{array}{l}4243.0 \\
4228.0\end{array}$} & $?$ & 0.5 : & 0.04: & 1: & \\
\hline & $?$ & $0.5:$ & 0.04: & 1: & \\
\hline 4101.7 & $\{\mathrm{H} \delta$ wings & 5.4 & 0.51 & $\left.\begin{array}{l}7 \\
5\end{array}\right\}$ & 26 \\
\hline \multirow{2}{*}{$\begin{array}{l}4076.2 \\
4068.6 \\
3970.1\end{array}$} & $\begin{array}{l}\mathrm{H} \delta \text { core } \\
{\left[\mathrm{S}_{\mathrm{II}}\right]}\end{array}$ & $\begin{array}{l}3.7 \\
2.3\end{array}$ & $\begin{array}{l}0.35 \\
0.22\end{array}$ & $\begin{array}{l}5 \\
3\end{array}$ & \\
\hline & [S II] & 3.5 & 0.34 & $5\}$ & 16 \\
\hline \multirow{2}{*}{$\begin{array}{l}3970.1 \\
3968.5\end{array}$} & $\mathbf{H} \varepsilon \quad$ ? & 10.3 & 1.03 & 14 & 52 \\
\hline & [Ne III] & & & & \\
\hline $\begin{array}{l}3889.1 \\
3888.6\end{array}$ & $\left.\begin{array}{l}\mathrm{H} \zeta \\
\mathrm{He}_{\mathrm{I}}\end{array}\right\}$ & 4.3 & 0.47 & 6 & 20 \\
\hline \multirow{2}{*}{$\begin{array}{l}3869.7 \\
3728.9\end{array}$} & [Ne III] & 19.3 & 2.12 & 29 & 120 \\
\hline & {$\left[\begin{array}{ll}\mathrm{O} & \mathrm{II}\end{array}\right]$ ) } & 28.7 & 3.75 & 51 & 35 \\
\hline $\begin{array}{l}3726.2 \\
3425.8\end{array}$ & $\begin{array}{l}{[\mathrm{O} \text { II }]} \\
{[\mathrm{Ne} \text { V] }}\end{array}$ & 14.5 & 2.46 & 33 & 130 \\
\hline & & & & & \\
\hline
\end{tabular}


lines arise in a dense region in which $N_{e} \geqslant 10^{8} \mathrm{~cm}^{-3}$. It is likely that the broad lines are produced by mass motions rather than by electron scattering and that this region is about $0.1 \mathrm{pc}$ in diameter. The mass of dense gas is only about $50 M_{\odot}$. A central mass of about $10^{8} M_{\odot}$ is required to retain this gas if it moves with a virial velocity of about $3000 \mathrm{~km} \mathrm{~s}^{-1}$, corresponding to the widths of the Balmer line wings.

(b) most of the low ionization forbidden lines together with the cores of the forbidden lines are produced in a gas with $T_{e} \simeq 20000 \mathrm{~K}$ and $N_{e} \simeq 5000 \mathrm{~cm}^{-3}$ which extends over a region about $50 \mathrm{pc}$ in diameter. This region, like the dense region cannot be completely filled, and the gas in it may be composed of clouds or filaments which occupy only about $\frac{1}{100}$ of the volume. This outer component has a mass of about $10^{4}$ $M_{\odot}$ and is moving at speeds of around $400 \mathrm{~km} \mathrm{~s}^{-1}$. Its total kinetic energy is about $4 \times 10^{52} \mathrm{erg}$.

(c) The source of the non-thermal continuum was shown by measurements from Stratoscope (Danielson et al., 1968) to be less than 0.1 or 5 pc in size; the timescale of the optical variations indicate that this region is still smaller, perhaps $10^{16} \mathrm{~cm}$ in diameter.

The physical processes which are going on in the nucleus of NGC 4151 are still obscure and the X-ray observations are obviously an important new source of information. The main problem is how the gas in the nucleus is ionized and stirred up. Several alternative ideas have been proposed to explain the overall spectrum of NGC 4151; these are summarized by Osterbrock (1971). One idea is that the gas is ionized by the non-thermal continuum. Nüssbaumer and Osterbrock (1970) showed that the observed strengths of the various ionization stages of $\mathrm{Fe}$ up to [ $\mathrm{Fe} \mathrm{X}$ ] could be explained in this way if the optical continuum $f_{v} \propto v^{-1.2}$ can be extrapolated below $234 \mathrm{eV}$, the ionization potential of Fe IX. A second possibility is that collisions between moving clouds or filaments produce a shock-heated gas with a temperature of several million degrees and that the thermal bremsstrahlung from this source in turn heats the remainder of the gas. This view of the nucleus of NGC 4151 as a chaotic assembly of moving and colliding gas clouds seems an attractive way of explaining the wide range of ionization conditions observed in NGC 4151 but it does not explain the ultimate source of the energy. The kinetic energy of the moving clouds would only last a few hundred years, less than the time taken for a typical cloud to cross the nucleus, at the present rate of radiative loss. Hence the problem of what stirs up the clouds is important.

The X-ray flux from the nucleus of NGC 4151 presumably arises either from the non-thermal source at the center or from the hot gas which appears to permeate the whole nucleus. In deciding between these two possibilities, the observed low energy cutoff in the X-ray spectrum is of great interest. This indicates a large column density (of order $10^{23}$ hydrogen atoms $\mathrm{cm}^{-2}$ ) which, as in the case of NGC 5128, could hardly be encountered in the outer parts of NGC 4151. Using the numbers given earlier, the low density $\left(N_{e}=5000 \mathrm{~cm}^{-3}\right)$ gas in the nucleus should present a typical cross section of about $4 \times 10^{21}$ atoms $\mathrm{cm}^{-2}$, while the high density $\left(N_{e} \simeq 10^{8} \mathrm{~cm}^{-3}\right)$ should present a cross section of about $3 \times 10^{23} \mathrm{~cm}^{-2}$, very similar to that given by 
the X-ray absorption. Now it is likely that the high density gas is responsible for weak, variable absorption lines seen at $\mathrm{He} \mathrm{I} \lambda 3889$ and in the blue wings of the lower Balmer lines (Anderson and Kraft, 1969). These absorption lines vary on a timescale of less than a year and this may be due to dense filaments or clouds moving across the continuum source (Rees and Sargent, 1972). In any case it seems likely that the X-rays come from the very center of NGC 4151 and not from the whole extended region that emits the forbidden line spectrum. A test of this hypothesis would be to look for variations in the X-ray flux and for variations in the amount of low energy absorption. Both should occur on timescales of several months.

\section{C. $3 \mathrm{C} 273$}

This is still the brightest known quasi-stellar object and consists of a 12th magnitude starlike object which radiates a spectrum very similar to that of a Seyfert galaxy, together with a faint jet about 20" long. The optical spectrum of 3C 273 contains very broad emission lines of $\mathrm{Mg}$ II, $\mathrm{H},[\mathrm{O}$ III $]$ and Fe II. There are no emission lines indicating the presence of a very hot gas in the spectrum of $3 \mathrm{C} 273$; however, the lines are much broader than those in NGC 4151 and weak features are correspondingly harder to detect. The optical continuous spectrum of $3 \mathrm{C} 273$ appears to be flat and fairly satisfactory models to explain the emission line spectrum have been achieved by extrapolating a spectrum $f_{v} \sim v^{-0.7}$ into the far ultraviolet (Bahcall and Kozlovsky, 1969). Their model has $N_{e} \simeq 3 \times 10^{7} \mathrm{~cm}^{-3}, T_{e}=1.7 \times 10^{4} \mathrm{~K}$, a radius $R=14 \mathrm{pc}$ and a filling factor $f=10^{-3}$. The mass of gas is $10^{5} M_{\odot}$. Osterbrock (1971) used this model to calculate the strengths of a number of emission lines and found that these agreed well with the observed line strengths in a typical QSO spectrum (see Table III). We thus have some confidence in Bahcall and Kozlovsky's model of $3 \mathrm{C} 273$; according to it the column density in an average direction through the gas is about $R f N_{e}=10^{24}$ hydrogen atoms $\mathrm{cm}^{-2}$. This is greater than the column density of $5 \times 10^{22} \mathrm{~cm}^{-2}$ inferred from the UHURU observation of a low energy

TABLE III

Comparison of $3 \mathrm{C} 273$ model with mean observed quasar spectrum

\begin{tabular}{|c|c|c|c|c|c|c|c|}
\hline Line & & $\begin{array}{l}\text { Relative } \\
\text { photon rate }\end{array}$ & $\begin{array}{l}\text { Observed } \\
\text { photon rate }\end{array}$ & Line & & $\begin{array}{l}\text { Relative } \\
\text { photon rate }\end{array}$ & $\begin{array}{l}\text { Observed } \\
\text { photon rate }\end{array}$ \\
\hline $\mathbf{L} \alpha$ & $\lambda 1216$ & 166.0 & $\mathbf{S}$ & Mg II & $\lambda 2799$ & 3.6 & $\mathbf{S}$ \\
\hline Civ & $\lambda 1549$ & 42.0 & $\mathbf{S}$ & $\mathrm{N} \mathrm{v}$ & $\lambda 1240$ & 2.8 & - \\
\hline He II & $\lambda 1640$ & 22.0 & W & [Ne v] & $\lambda 3426$ & 2.4 & W \\
\hline $\mathbf{H} \beta$ & $\lambda 4861$ & 20.0 & $\mathbf{M}$ & O III] & $\lambda 1664$ & 2.1 & - \\
\hline He II & $\lambda 4686$ & 18.0 & - & Si IV & $\lambda 1397$ & 1.7 & - \\
\hline $\mathbf{H} \gamma$ & $\lambda 4340$ & 10.0 & $\mathbf{M}$ & [O III] & $\lambda 5007$ & 1.6 & $\mathbf{M}$ \\
\hline C III ] & $\lambda 1909$ & 9.4 & $\mathbf{M}$ & Si III] & $\lambda 1892$ & 1.2 & - \\
\hline C II] & $\lambda 2327$ & 8.0 & obs & [Mg VII] & $\lambda 2632$ & 1.1 & - \\
\hline O IV] & $\lambda 1402$ & 6.0 & $\mathbf{M}$ & [O III] & $\lambda 4363$ & 1.0 & obs \\
\hline $\mathbf{H} \delta$ & $\lambda 4101$ & 6.0 & obs & [Ne v] & $\lambda 3346$ & 0.9 & obs \\
\hline $\mathrm{O}$ VI & $\lambda 1034$ & 5.6 & - & & & & \\
\hline
\end{tabular}


cutoff in the X-ray spectrum. There are several possible reasons for the discrepancy. The elements $\mathrm{Ne}, \mathrm{O}$ and $\mathrm{C}$ which are chiefly responsible for the $\mathrm{X}$-ray absorption, are completely ionized for an appreciable fraction of the radius in these models. Moreover, the filling factor $f$ is only a crude average way of describing what is probably a very inhomogeneous situation so that $f$ could be appreciably smaller in the direction of the line of sight than it is on average.

\section{X-Ray Sources in Clusters of Galaxies}

There is preliminary evidence that the X-ray sources in clusters are all extended; the X-ray clusters may all contain radio galaxies in which case their X-ray emission is more likely to be non-thermal rather than thermal bremsstrahlung from a hot gas in the cluster. We shall concentrate our discussion on the nearby clusters - Virgo, Perseus, Coma and Centaurus - that are known to be extended at the time of this Symposium.

\section{A. THE VIRGO CLUSTER}

This is the nearest reasonably populous cluster of galaxies. According to de Vaucouleurs (1961), The Virgo cluster consists of a diffuse elliptical cloud of spiral and irregular galaxies centered at $12^{\mathrm{h}} 27^{\mathrm{m}} \cdot 5,+13.9$ and a concentrated cluster of elliptical and SO galaxies centered at $12^{\mathrm{h}} 26^{\mathrm{m}} \cdot 5,+13.2$. The distribution of bright galaxies over the sky near the Virgo cluster according to morphological type is shown in Figure 3, which is taken from de Vaucouleurs' paper. The mean redshift of the E cloud is $+950 \mathrm{~km} \mathrm{~s}^{-1}$ and that of the $S$ cloud $+1450 \mathrm{~km} \mathrm{~s}^{-1}$.

The X-ray source in the Virgo cluster is centered on M $87 \equiv$ NGC 4486 , the famous radio galaxy with the remarkable blue jet. This galaxy lies at $12^{\mathrm{h}} 28^{\mathrm{m}} \cdot 3,+12^{\circ} 40^{\prime}$ and is not quite at the center of the $\mathrm{E}$ cloud. The dense core of the $\mathrm{E}$ cloud contains 19 bright ellipticals within a radius of $1: 25$ from the center; around this core the elliptical galaxies are distributed very unevenly. The $\mathrm{E}$ cloud in the Virgo cluster has a radial velocity dispersion of $550 \mathrm{~km} \mathrm{~s}^{-1}$; this leads to an estimated mass for the system of about $4 \times 10^{14} M_{\odot}$ and a mean mass per galaxy of around $4 \times 10^{12} M_{\odot}$. This exceeds by at least an order of magnitude the mass estimated to be in the galaxies.

\section{B. THE PERSEUS CLUSTER}

The Perseus cluster, shown in Figure 4, is one of the nearest clusters of galaxies to qualify for entry in Abell's (1958) catalogue of rich clusters of galaxies. (Table IV lists all clusters of distance class 2 or less in Abell's catalogue). The Perseus cluster is very irregular in shape. The densest concentration of galaxies is around NGC 1275, the radio galaxy 3C 84 which is also a Seyfert galaxy. From NGC 1275 a chain of galaxies stretches to the west, terminating at IC 310 which is also a weak radio galaxy. Most of the galaxies in the Perseus cluster are ellipticals and SO galaxies. Recent studies of the dynamics of the Perseus cluster by Chincarini and Rood (1971) and by Gunn and Sargent (1972) have shown several peculiar features. The radial 


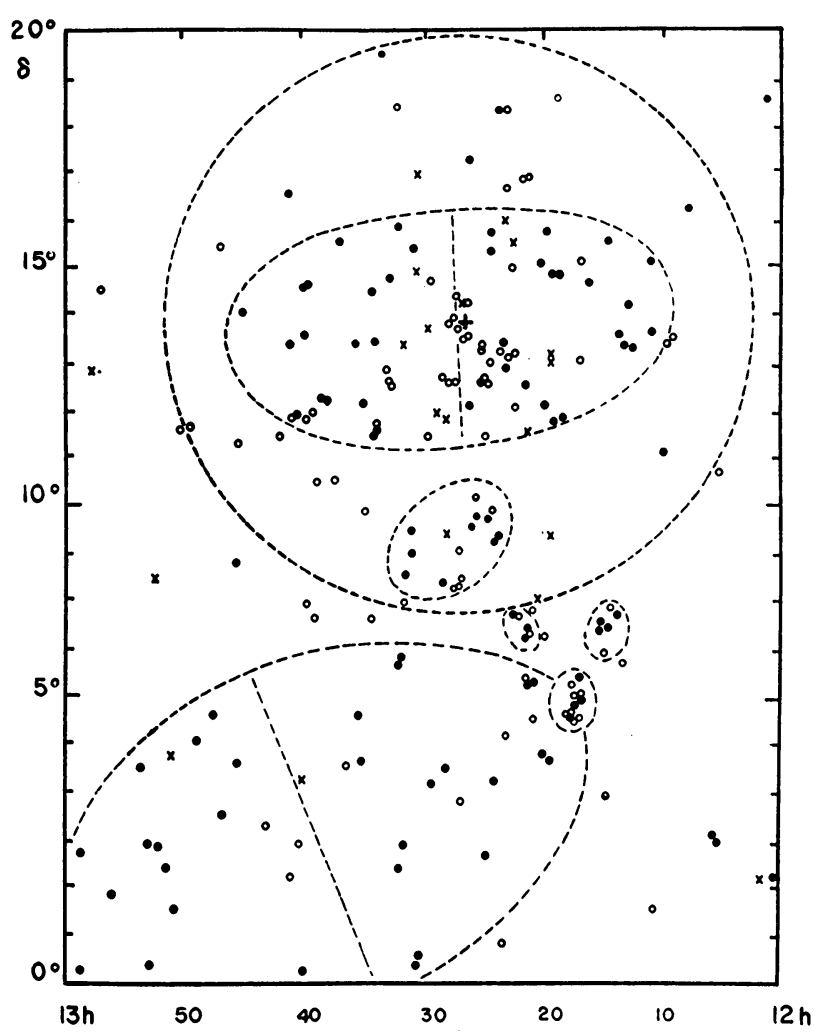

Fig. 3. Distribution of 212 galaxies in the region of the Virgo Cluster. Dots: spirals and irregulars; circles: ellipticals and lenticulars; crosses: type unknown. (From de Vaucouleurs, 1961).

velocity dispersion of the cluster is $1420 \mathrm{~km} \mathrm{~s}^{-1}$ about its mean redshift of 5460 $\mathrm{km} \mathrm{s}^{-1}$. This is the largest velocity dispersion of any known cluster and leads to a mass estimate from the virial theorem of about $10^{15} M_{\odot}$. Since the cluster contains at most 50 large galaxies, either most of this mass is in dark matter or the cluster is unstable on a timescale of about $3 \times 10^{8} \mathrm{yr}$. Despite the existence of an obvious chain of galaxies in the Perseus cluster, there is no evidence that the system is rotating; moreover, there is no obvious correlation of redshift with position in the cluster. Although Chincarini and Rood (1971) fitted a single Gaussian curve to the histogram of a number of galaxies versus redshift, there is some evidence in their data, as well as in that of Gunn and Sargent, (1972) that the distribution might be bimodal. This might indicate that Perseus, like Virgo, is in reality two clusters superimposed on the line of sight; more redshifts are being obtained in order to study this possibility further.

NGC 1275, whose redshift is close to that of cluster mean, is remarkable in that, as well as containing a Seyfert nucleus, it has outer emission patches which are receding from it at $3000 \mathrm{~km} \mathrm{~s}^{-1}$. Lynds (1970) has published a beautiful photograph showing that this receding nebulosity has a filamentary structure, similar to that in the Crab nebula. 


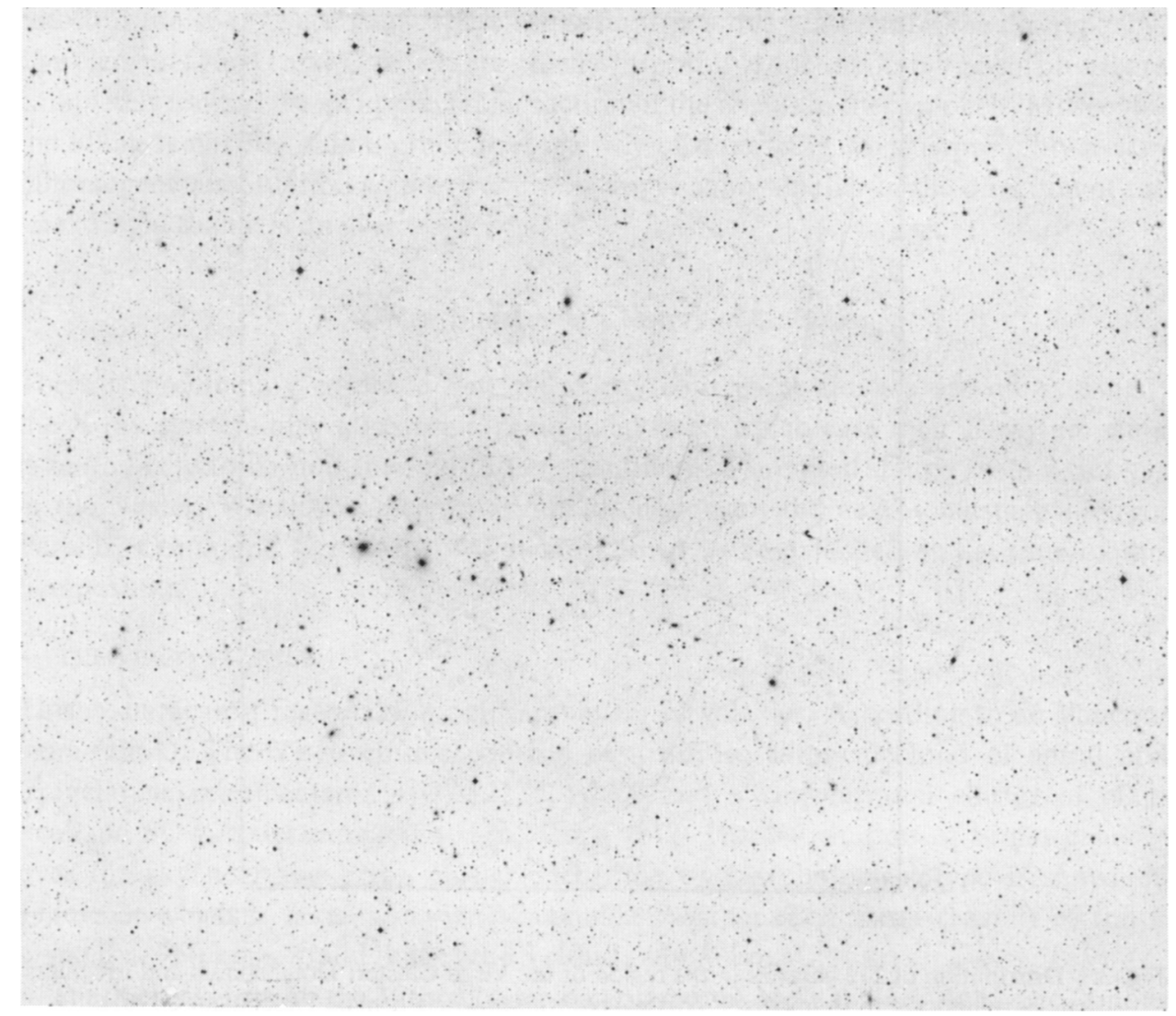

Fig. 4. Perseus Cluster of Galaxies (Hale Observatories photo). North is up and east is to the left.

Radio observations by Ryle and Windram (1968) show possible evidence for a physical interaction between NGC 1275 and IC 310 to the W and NGC 1265, the radio galaxy $3 \mathrm{C} 83.1$, which lies $27^{\prime}$ to the $\mathrm{NW}$ of $\mathrm{NGC} 1275$. The radio contours around IC 310 and NGC 1265 are in each case bent away from the direction to NGC 1275 in the form of a tail; moreover, the radio center is in each case displaced from the center of the optical galaxy away from NGC 1275. This evidence for interactions between remote galaxies is particularly remarkable because NGC 1265 has a redshift which is $2370 \mathrm{~km} \mathrm{~s}^{-1}$ greater than that of NGC 1275 and the center of the Perseus cluster. A mass of $10^{15} M_{\odot}$ for the cluster is again required to gravitationally bind NGC 1265. The observations by Ryle and Windram (1968) at $408 \mathrm{MHz}$ show that the Perseus cluster contains an extended source $3 \mathrm{C} 84 \mathrm{~B}$ which is centered between NGC 1275 and NGC 1265, unlike the extended source of X-ray emission which appears to be centered on NGC 1275.

C. THE COMA CLUSTER

This cluster, shown in Figure 5, is the prototype example of a spherical, centrally 
TABLE IV

Abell clusters nearer than distance class 2

\begin{tabular}{|c|c|c|c|c|c|c|}
\hline $\begin{array}{l}\text { Abell No. } \\
\text { (1) }\end{array}$ & $\begin{array}{l}D \\
(2)\end{array}$ & $\begin{array}{l}R \\
\text { (3) }\end{array}$ & $\begin{array}{l}\text { Magn. } \\
\text { (4) }\end{array}$ & $\begin{array}{l}\text { Class } \\
(5)\end{array}$ & $\begin{array}{l}\text { Radio } \\
\text { flux (6) }\end{array}$ & Remarks (7) \\
\hline 194 & 1 & $\mathbf{0}$ & 13.9 & II & 5.5 & $3 C 40$ \\
\hline 262 & 1 & $\mathbf{0}$ & 13.3 & III & 0.15 & \\
\hline 347 & 1 & $\mathbf{0}$ & 13.3 & II-III & 9.2 & $3 \mathrm{C} 66$ \\
\hline 400 & 1 & 1 & 13.9 & II-III & 6.3 & $3 C 75$ \\
\hline 407 & 2 & 0 & 14.7 & - & 0.7 & $4 C 35.6$ \\
\hline 426 & 0 & 2 & 12.5 & II-III & 13.0 & Perseus; 3C 83.1, 3C 84 \\
\hline 539 & 2 & 1 & 14.4 & III & 0.13 & \\
\hline 548 & 1 & 1 & 13.7 & III & 0.19 & \\
\hline 569 & 1 & 0 & 13.8 & - & 1.05 & Radio size $\sim 1.4$ \\
\hline 576 & 2 & 1 & 14.4 & III & $<0.1$ & \\
\hline 779 & 1 & 0 & 13.8 & II & 0.20 & \\
\hline 1060 & 0 & 1 & 12.7 & III & $<0.1$ & \\
\hline 1185 & 2 & 1 & 14.3 & - & $<0.1$ & \\
\hline 1213 & 2 & 1 & 14.5 & III & 1.9 & CTD 72 ; radio size $\sim 1^{\prime}$ \\
\hline 1228 & 1 & 1 & 13.8 & III & $<0.1$ & \\
\hline 1314 & 1 & 0 & 13.9 & - & 0.93 & \\
\hline 1367 & 1 & 2 & 13.5 & II-III & 5.90 & $3 \mathrm{C} 264$; radio size $\sim 1.5$ \\
\hline 1656 & 1 & 2 & 13.5 & II & 0.74 & Coma; (Coma C) \\
\hline 1736 & 2 & 0 & 14.8 & III & 0.64 & \\
\hline 2147 & 1 & 1 & 13.8 & - & 0.92 & Radio size $\sim 1 \prime 3$ \\
\hline 2151 & 1 & 2 & 13.8 & III & 1.2 & Hercules \\
\hline 2152 & 1 & 1 & 13.8 & III & $<0.1$ & \\
\hline 2162 & 1 & 0 & 13.7 & - & $<0.1$ & \\
\hline 2197 & 1 & 1 & 13.9 & Il & $<0.1$ & \\
\hline 2199 & 1 & 2 & 13.9 & I & $<3.55$ & NGC $6166 ; 3 C 338$ \\
\hline 2634 & 1 & 1 & 13.8 & I-II & 3.0 & NGC $7720 ; 3 C 465$ \\
\hline 2666 & 1 & 0 & 13.8 & - & $<0.1$ & \\
\hline
\end{tabular}

Notes: Column (2): distance class;

Column (3): richness class;

Column (4): magnitude of 10th brightest galaxy;

Column (5): morphological class of cluster from Bautz and Morgan (1970);

Column (6): radio power in flux units at $1415 \mathrm{MHz}$ from Fomalont and Rogstad (1966).

condensed rich cluster. Such clusters almost invariably contain predominantly E and SO galaxies. The Coma cluster is dominated by two very luminous galaxies, NGC 4889 to the E and NGC 4874 to the W. Counts show that the center of the cluster lies close to NGC 4874. A dynamical study of the Coma cluster has recently been made by Rood et al. (1972) on the basis of many new redshift measurements. The radial velocity dispersion of the cluster is $1060 \mathrm{~km} \mathrm{~s}^{-1}$ and the mean redshift is $6888 \mathrm{~km} \mathrm{~s}^{-1}$. The autors find that the cluster is not measurably rotating and that the bright and faint galaxies have the same dispersion in radial velocity within the accuracy of the data. They conclude that the highly condensed, spherical, shape of the cluster must indicate that it is stable. The derived mass from the virial theorem is $3.4 \times 10^{15} M_{\odot}$ and the mass to light ratio is $M / L_{p} \sim 250$. This is higher than that obtained for individual elliptical galaxies by a factor of around 5 . 
Fig. 5. Coma Cluster of Galaxies (Hale Observatories photo). North is up and east is to the left.

Weak radio sources have been observed at 408 and $1407 \mathrm{MHz}$ in the Coma cluster by Willson (1970). Thirteen are certainly or probably associated with individual Coma galaxies, including NGC 4874 but not its luminous companion NGC 4889. NGC 4869 which lies about $6^{\prime}$ to the SW of NGC 4874 is a weak source and Willson suggests from the shape of the radio contours that the two galaxies may be interacting. In addition to discrete sources, the Coma cluster has an extended, low frequency, radio halo about $40^{\prime}$ arc or $80 \mathrm{kpc}$ in diameter and at the center of the cluster. Willson supposes that this weak halo is associated in some way with NGC 4874 . He estimates that to maintain the halo would require the ejection of $10^{51} \mathrm{erg}^{-1}$ for the past $3.5 \times 10^{9}$ yr from NGC 4874 .

Although the Coma cluster is usually considered in the literature to be symmetric, there is a conspicuous chain or fan of galaxies extending to the SW from NGC 4874. This is obvious to the eye and persists on contours made from deep counts (Dam and Waddington 1972), as can be seen on Figure 6.

\section{THE CENTAURUS CLUSTER}

An extended X-ray source about $30^{\prime}$ in diameter and centered roughly on NGC 4696 has been discovered by UHURU. NGC 4696 is the radio source Pks 1245-41; it is 


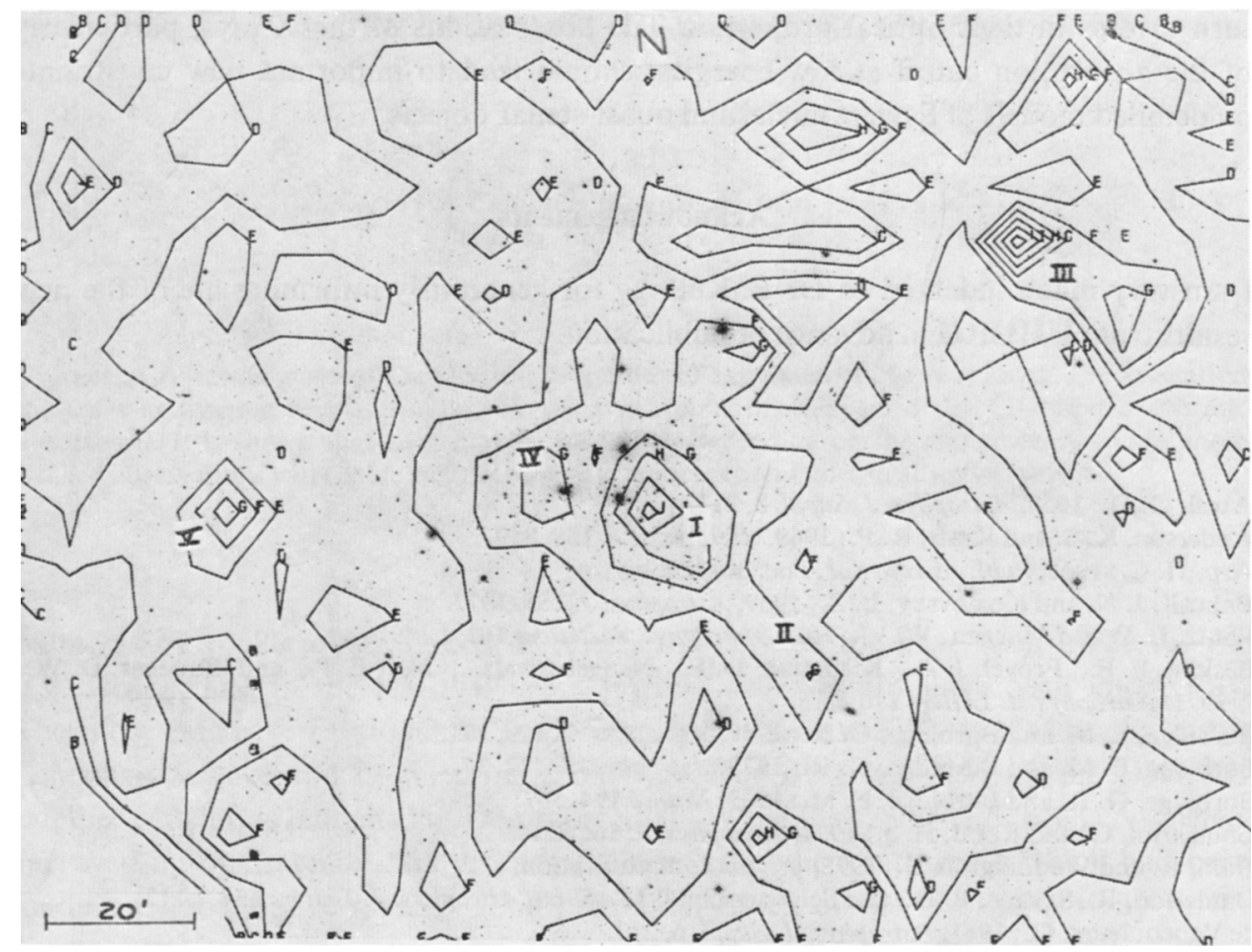

Fig. 6. Contour map of the Coma Cluster with spur extending from NGC 4875 (II). North is up and east is to the left.

an $\mathrm{E}$ galaxy in a rich cluster. Klemola (1969) states that the cluster contains 100 galaxies over an area $180^{\prime} \times 120^{\prime}$. Most of the galaxies in the cluster appear to be spirals and lenticulars. The redshift of NGC 4696 is $2790 \mathrm{~km} \mathrm{~s}^{-1}$ (Burbidge and Burbidge, 1972); its spectrum contains the normal absorption lines plus emission lines of $[\mathrm{N} \mathrm{II}]$ and $\mathrm{H} \alpha$. As is common in the nuclei of normal galaxies, $[\mathrm{N} \mathrm{II}]>\mathrm{H} \alpha$.

\section{Conclusions}

\section{A. CLUSTER SOURCES}

So far all the extended X-ray sources are associated with clusters of galaxies which contain a radio galaxy. In several cases the radio galaxy is surrounded by a weak radio halo. The clusters concerned have diverse shapes, degrees of central condensation and galactic populations. These observations point to a non-thermal origin - inverse Compton effect on the microwave background photons of the relativistic electrons responsible for the radio halos - rather than thermal bremsstrahlung resulting from infalling intergalactic matter (Gott and Gunn, 1971).

B. INDIVIDUAL SOURCES

These appear to be produced in the centers of active galactic nuclei which are in 
turn diverse in their optical properties. The observations of the X-rays, particularly of the absorption cutoff at low energies, should lead to important new constraints on detailed models of Seyfert nuclei and quasi-stellar objects.

\section{Acknowledgements}

I am very much indebted to Dr E. Kellogg for generously informing me of the new results from UHURU in advance of publication.

\section{References}

Abell, G. O.: 1958, Astrophys. J. Suppl. 3, 211.

Anderson, K. S. and Kraft, R. P.: 1969, Astrophys. J. 158, 859.

Arp, H. C.: 1968, Publ. Astron. Soc. Pac. 80, 129.

Bahcall, J. N. and Kozlovsky, B. Z.: 1969, Astrophys. J. 155, 1077.

Bautz, L. P. and Morgan, W. W.: 1970, Astrophys. J. Letters 162, L149.

Becklin, E. E., Frogel, J. A., Kleinman, D. E., Neugebauer, G., Ney, E. P., and Strecker, D. W.: 1971, Astrophys. J. Letters 170, L15.

Burbidge, E. M. and Burbidge, G. R.: 1959, Astrophys. J. 129, 271.

Burbidge, E. M. and Burbidge, G. R.: 1972, Astrophys. J. 172, 37.

Burbidge, G. R. and Burbidge, E. M.: 1962, Nature 194, 367.

Chincarini, G. and Rood, H. J.: 1971, Astrophys. J. 16., 321.

Dam, R. and Waddington, B.: 1972, private communication.

Danielson, R., Savage, B. D., and Schwarzschild, M.: 1968, Astrophys. J. Letters 154, L117.

de Vaucouleurs, G.: 1961, Astrophys. J Suppl. 6, 213.

de Vaucouleurs, G.: 1968, Nearby Groups of Galaxies, preprint.

Fomalont, E. B. and Rogstad, D. H.: 1966, Astrophys. J. 146, 528.

Gott, J. Richard, III and Gunn, James E.: 1971, Astrophys. J. Letters 169, L13.

Gunn, J. E. and Sargent, W. L. W.: 1972, in preparation.

Klemola, A. R.: 1969, Astron. J. 74, 809.

Kunkel, W. E. and Bradt, H. V.: 1971, Astrophys. J. Letters 170, L7.

Lynds, C. R.: 1970, Astrophys. J. Letters 159, L151.

Mathews, T. A., Morgan, W. W., and Schmidt, M.: 1964, Astrophys. J. 140, 35.

Nüssbaumer, H. and Osterbrock, D. E.: 1970, Astrophys. J. 161, 811.

Oke, J. B. and Sargent, W. L. W.: 1968, Astrophys. J. 151, 807.

Osterbrock, D. E.: 1971, in D. J. K. O'Connell (ed.), The Nuclei of Galaxies, North-Holland Publ. Co, Amsterdam, p. 151.

Rees, M. J. and Sargent, W. L. W.: 1972, Comm. Astrophys. Space Sci.4, 7.

Rood, H. J., Page, T. L., Kintner, E. C., and King, I. R.: 1972, Astrophys. J. 175, 627.

Ryle, M. and Windram, M. D.: 1968, Monthly Notices Roy. Astron. Soc. 138, 1.

Wade, C. M.: 1968, Astron. J. 73, 876.

Wade, C. M., Hjellming, R. M., Kellermann, K. I., and Wardle, J. F. C.: 1971, Astrophys. J. Letters 170, L11.

Weedman, D. W.: 1971, Astrophys. J. Letters 167, L23.

Willson, M. A. G.: 1970, Monthly Notices Roy. Astron. Soc. 151, 1. 